\title{
Recognition of emotions through facial expression analysis
}

DOI: $10.46932 / s f j d v 2 n 2-076$

Received in: March 1st, 2021

Accepted in: May 30th, 2021

Freddy Alejandro Castro Salinas

Máxima formación académica: Psicólogo clínico.

Institución: Unidad de Posgrados de la Universidad Católica de Cuenca.

Dirección laboral: Ecuador, Azuay, Gualaceo, Fidel Antonio Piedra entre Cuenca y Colón 5-46.

Correo electrónico: alejandrocastro2295@gmail.com

\section{Geovanny Genaro Reivan Ortiz}

PhD en Psicología mención "metodología y estadística"

Institución: Laboratorio de Psicología Básica, análisis conductual y desarrollo programático.

Universidad Católica de Cuenca, Campus CIITT.

Dirección personal: Ecuador, Azuay, Cuenca, parroquia Ricaurte, Vía Ricaurte Bibín frente a Plásticos Rival.

Correo electrónico: greivano@ucacue.edu.ec

\section{Pedro Carlos Martínez Suarez}

Máxima formación académica: PhD en Psicología.

Institución: Laboratorio de Psicometría, etología y Psicología comparada. Universidad Católica de Cuenca, Campus CIITT.

Dirección personal: Ecuador, Azuay, Cuenca, parroquia Ricaurte, Vía Ricaurte Bibín frente a Plásticos Rival.

Correo electrónico: pmartinezs@ ucacue.edu.ec

\begin{abstract}
The possibility of recognizing what emotion one of our peers is experiencing has been the subject of study by various researchers over the years, Paul Ekman being the one who has delved most deeply into this subject, the most viable and simple way to achieve this would be through the analysis of people's facial expressions. The search for information was carried out using rigorous exclusion criteria such as studies corresponding to grizzly data and letters to the editor, and inclusion criteria such as studies published only in high impact journals such as PubMed, Elsevier, Taylor \& Francis, ScienceDirect, APA PsycNet and Springer, PRISMA guidelines and AMSTAR check-list were used. The main objective of this systematic review was to determine whether there is sufficient scientific literature evidence to clarify whether it is possible to accurately identify the six basic universal emotions "happiness, surprise, sadness, anger, fear and disgust" proposed by Paul Ekman through facial expressions. After the analysis of the articles collected and based on the main findings, it is concluded that the recognition of emotions through facial expressions is a subject that still needs to be studied in greater depth, as suggested by the results obtained.
\end{abstract}

Keywords: Facial expression, basic emotions, Paul Ekman. 


\section{INTRODUCTION}

The study of emotions in recent years has been increasing in a formidable way reaching an increasing degree of relevance in the field of research as a result of this it has been identified that about 250 scientists are working based on this theory (Ekman, 2016), within this general theme there is a specific topic on which we base our research, "the possibility of recognising emotions by analysing facial expression", a hypothesis that generates interest and debate in the scientific community because there are researchers who support and endorse this theory as (Sato et al. 2019; Namba et al, 2018 \& Calvo et al., 2016) and others who show their scepticism in believing that this is possible to do as (Naab et al., 2017; Jack et al., 2012 \& Samara et al., 2017).

Throughout history, different authors have wanted to determine the role that emotions play in the life of human beings; as well as to establish a specific guide to identify and interpret them correctly through facial expressions, however, until now this has not been achieved, because the authors who worked on this topic mostly used different procedures, methodologies, variables and materials during their research process, so that at the end of their work they obtained dissimilar conclusions each of them (Ekman, 2016).

In the 19th century (Darwin, 1873) in his book "The Expression of the Emotions in Man and Animals", he argues that emotional expressions were created as part of an adaptive evolution in living beings over the years, also indicating that these facial expressions triggered by an emotion have the main function of "communicating" to their peer's relevant information that can serve them for survival or to expose to others their current mood (Hess et al, 2009).

(Park et al., 2017), point out the six basic universal emotions that were defined by (Ekman \& Friesen, 1971), "happiness, surprise, anger, fear, disgust and sadness" or main as some authors call them, it is worth mentioning that some authors/articles often place synonyms for some emotions instead of placing the word written by Ekman.

On the other hand (Ekman, 1972), refers that the six basic universal emotions are innate and transcultural so they can be identified in any part of the world and in any person regardless of their place of origin, this would be achieved thanks to the fact that each of these emotions generate their own distinctive features and different perceptual facial movements in each individual (Blandon et al., (2017).

The importance of this topic for psychology lies in many aspects, however, the hypothesis that suggests that an individual's failure to recognize emotions in his or her peers may be indicative of some kind of psychological disturbance or problem, or be a symptom of a psychiatric pathology, is the most striking and should be studied in greater depth (Csukly et al., 2008). 
The elaboration of this article will also allow its reader to gain knowledge about the existence of different variables that could modify and influence the results obtained after studying the recognition of emotions through facial expression such as: the types of "dynamic or static" materials that were used during the investigations (Calvo et al., 2016); the technology used to conduct the studies (Stockli et al. , 2017); the age of the study samples (Ruffman et al. , 2008); the gender of the people (Donges et al. , 2012); the consumption of drugs "alcohol" (Foisy et al. , 2007); the presence of diseases at a cognitive level (Elferink et al. , 2015), among others.

Following the theory, the question arises: Are there universal emotions that represent facial features? E.g. Due to the inconsistency of studies on the subject, the present study aims to analyses the recognition of emotions (happiness, disgust, anger, sadness, surprise and fear "Paul Ekman") through facial features by means of a systematic review of scientific articles, which will contribute to the clarification of the gaps in knowledge about Ekman's studies on facial expressions; Theoretically contrast universal emotions through facial features; identify variables that affect the correct recognition of emotions through facial expressions; and determine whether the emotions of happiness, disgust, anger, sadness, surprise and fear in empirical articles refer to facial features.

\section{METHOD:}

\subsection{DESIGN}

Systematic (desk) review.

\subsection{WRITE OPERATION}

The quality of the study presentation was based on the AMSTAR check-list, a tool to assess the quality of the review (see Annexed 1) and the PRISMA guidelines "systematic review reporting" (see Annexed 2). The review protocol was developed following the procedures of the Cochrane Handbook for Systematic Reviews.

\subsection{INCLUSION CRITERIA}

Studies with cross-sectional and longitudinal empirical methodology; prospective and retrospective studies; articles from 2000 onwards; peer-reviewed from high impact journals; articles published in English; studies with a larger study population of young adults ( $>18$ years).

\section{Exclusion criteria}

Studies that correspond to grizzy data, letters to the editor, case study analysis; and studies that have not been externally refereed. 


\subsection{PROCEDURE AND ELIGIBILITY OF ARTICLES}

Advanced searches were carried out using thesaurus commands (AND, OR, +, -, ALLINTITLE, ALLINTEXT, FYLETYPEII), in the following databases: PubMed, Elsevier, Taylor \& Francis, ScienceDirect, APA PsycNet and Springer of empirical articles since 2000; by means of the keywords: "facial expression", "basic emotions" and "Paul Ekman".

\section{Article selection and data entry}

The lead author examined the eligibility of the articles by reading the abstract and key words, determining the suitability of the study by the protocols mentioned; data extraction will be done by means of a table and a flow chart with the most substantial characteristics of the research (year, author, objective, method, aspect discussed, database). The quality of the studies was analysed using the NICE tool for contrasting quality empirical articles. A total of 133 articles were obtained from the scientific database search, of which 36 were eliminated because they did not meet the inclusion criteria, 4 because they were duplicates and 29 because they did not contain information related to the topic. A total of 64 articles were used for this systematic review (see Figure 1).

Figure 1: Information flow

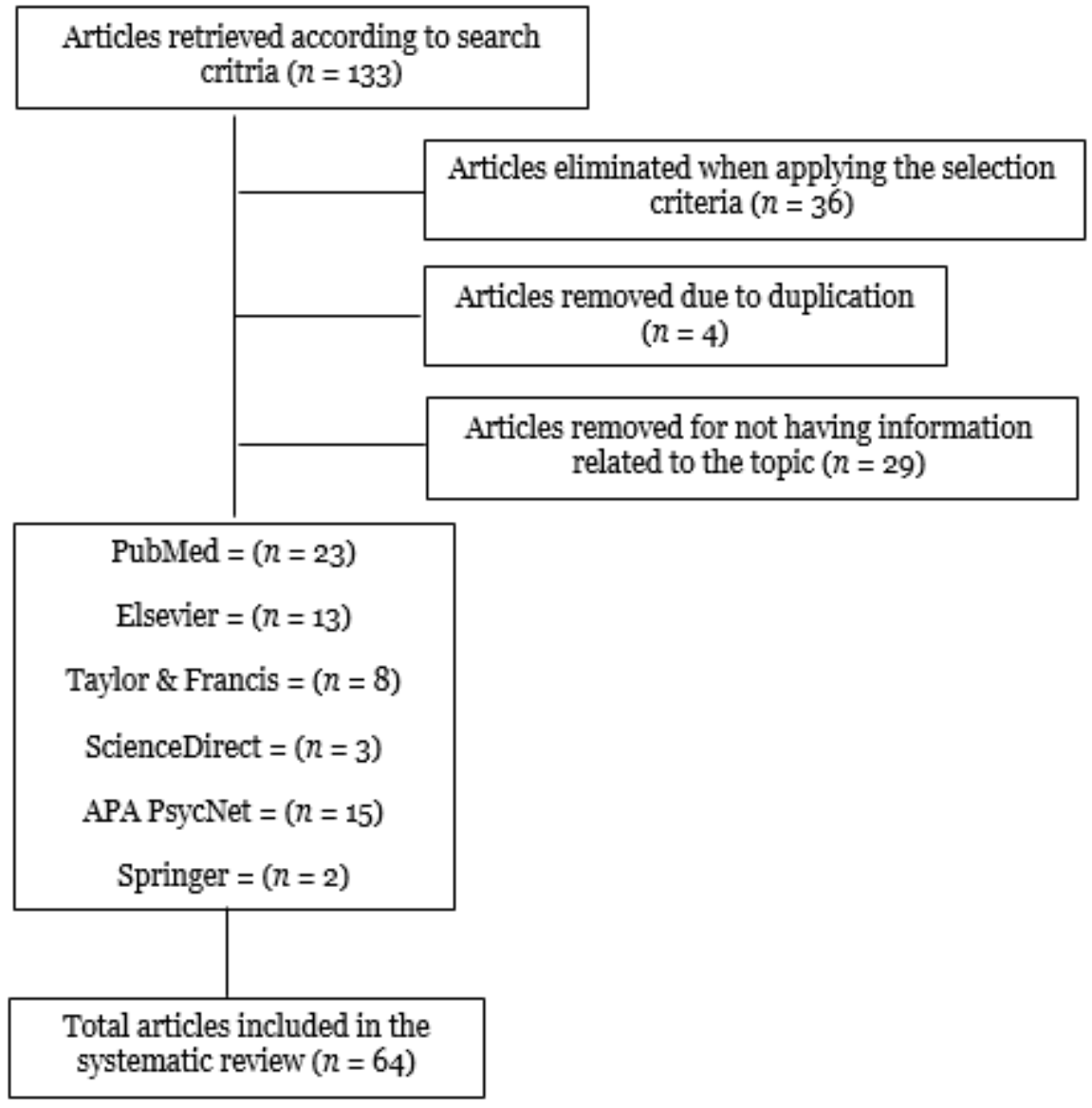




\section{RESULTS:}

According to the relevant study after searching for information in databases of empirical articles, the evidence collected indicates that research such as (Naab et al., 2007), determines that the possibility of identifying emotions through the analysis of facial expression does not reach the level of recognition indicated by Paul Ekman in (1980), it should be noted that these authors used the same photographs taken, coded and labelled by Ekman and Frisen in (1976).

On the other hand, the conception of a universal emotional language is challenged, (Jack et al., 2012), determine that culture can modify the facial expressions that people exhibit when feeling an emotion, an idea shared by (Mishra et al., 2018), who point out the existence of subtle but significant cross-cultural differences when correctly classifying emotions, following this same line (Barrett et al., 2019), remarking that the way of communicating an emotion varies significantly depending on the culture or situation in which the individual is.

They point out (Samara et al., 2017), that an individual's emotional states cannot be accurately identified by means of their facial expressions since during an active interaction there is greater facial variation for an emotion than when the same emotion is in a passive state. (Tarnowski et al., 2017), point out that a person's facial expression can be modified whether the emotion displayed and experienced by the subject is real or acted out.

On the other hand, (Said et al., 2009), together with his team, generated the hypothesis that the features of a person's face may resemble the facial expressions generated by an emotion, which would create confusion and errors when trying to identify them.

It also shows that the direction of a person's gaze does not influence the recognition of the emotion fear as has been reported in recent years, regardless of the age of the subject (Slessor et al., 2010). Similarly, it is suggested that more studies are needed to establish a direct and shared relationship between gaze direction as a facial trait and the detection of the emotion sadness (Semyonoy et al., 2019), but these last two conclusions are challenged by (Adams et al., 2005), who argue that gaze direction as a facial expression does help to more easily determine the interpretation of an emotion, whether it is positive or negative.

At the end of their research study and based on the results obtained (Chóliz et al., 2012), they comment that the pressure in the recognition of emotions through facial expression varies significantly between the emotion that was exposed in the photograph and the responses that the study sample was able to provide after identifying them.

Regarding the possibility of recognising emotions by technological means, authors such as (Stöckli et al., 2017), describe that the technology gives very different results depending on the type of variable 
that they decipher, when they read static prototypical images their level of assertiveness is high, but when analysing a real emotion displayed on a human face their level of assertiveness drops considerably; However, within the literature analysed, it was found that an algorithm based on recognising and capturing emotional expressions in real time both in 2D and 3D through Kinect sensors has been developed that shows better results compared to other software identifying emotions through facial expressions (Mao et al., 2015).

It is argued that the person's own age can generate some confusion when correctly identifying an emotion, because old faces tend to generate in people the perception of enunciating happiness, although this is not exactly so, adding that the wrinkles that are appearing typical of an older age can also cause mistakes to be made when performing this task (Hass et al., 2015).

On the other hand (Gosselin et al., 2010), culminate their research by stating that the facial expressions exhibited by the voluntary control group after imitating the emotions presented to them were clearly better in certain action units, managing to activate expected muscles such as: wrinkling of the forehead, jaw drop, eyebrow elevation, lifting of the lower lip, etc., while in other action units there was not so much agreement as they did not manage to activate the required muscles such as: the external and internal elevators of the eyebrows or the depressors of the corners of the mouth or stretching of the lips, etc.

Authors such as (Rohr et al., 2012), who support the validity of this theory, refer that the recognition of emotions through the analysis of facial expression from one individual to another can even occur unconsciously, especially when discerning between positive and negative emotions. For their part (Sato et al., 2019), mention that it is possible that there is a universal emotional language that can be identified through facial expressions mainly for the six basic emotions described by Ekman, but refer that for this theory to be validated and accepted more solid empirical evidence is needed.

It is noted that emotions can be recognised more quickly and accurately through facial expressions when dynamic rather than static materials are used, as the former facilitate the interpretation of emotions, whereas when using the latter the degree of assertiveness decreases considerably (Namba et al., 2018), a conclusion supported by (Biele et al., 2006 \& Calvo et al., 2016).

Several authors also share the idea that the easiest emotion to recognise is happiness (Calvo et al., 2016; Maher et al., 2014; Huang et al., 2012 \& Montagne et al, 2007), Maher points out that this could be due to the fact that in the facial expression generated by happiness there is a lifting of the lower lip, which makes it easier to identify, while Haung and his team report that the most complex emotions to identify are surprise and sadness, an idea that is countered by Montagne and his colleagues who allude that fear is the most complicated emotion to identify. 
It has been proposed that training to recognise emotions is possible and that this instruction is not only useful to improve the recognition of the trained emotion but also helps in the identification of other emotions, especially happiness, adding that to improve accuracy in recognising sadness the only way is through the instruction of this same emotion, another idea that emerges from this research is that there is a tendency to confuse disgust with anger and fear with surprise (Wang et al., 2018).

In the face of new hypotheses that have emerged on this subject (Horstmann, 2003), he concludes that facial expressions communicate emotions, whether positive or negative, ruling out that they indicate requests for behaviour or postulations for action, as other theories suggest.

On the other hand (Douglas et al., 2012), propose that people can more easily and assertively identify emotions through facial expressions when they have knowledge about specific landmarks to observe on the face compared to when they must do so by paying attention to the whole face of the individual, for example: observing only the smiling mouth of a subject instead of the whole face provides better results in identifying the emotion happiness.

This systematic review also provided information on how some pathologies influence the correct identification of emotions through facial expressions, as indicated by (Cortez et al., 2017), who state that people suffering from ADHD identify basic universal emotions more quickly but do so with little precision, the authors point out that this could be due to the impulsivity of this pathology to which these individuals are exposed.

On the other hand (Domes et al., 2009), conclude that individuals with borderline personality disorder compared to a healthy control group, as a result of their emotional lability make more mistakes when recognizing emotions correctly through facial expressions, however this statement is refuted by (Unoka et al., 2011), who comment that people with this pathology make mistakes only when identifying negative emotions but not positive ones, while (Dyck et al., 2008), state that individuals with BPD do not demonstrate deficiencies in identifying emotions compared to their healthy peers when the time to do so is unlimited, however, when these subjects are exposed to a time limit to perform this task they fail considerably.

While (Ricciardi et al., 2017), indicate that Parkinson's disease generates in people who suffer from it difficulty in both identifying emotions in others and replicating emotions correctly, this is due to the fact that specific areas responsible for these functions at the neurological level are impaired.

Following this same premise (Monti et al., 2015), they point out that people with temporal lobe epilepsy show difficulties in assertively identifying the negative emotions of anger, sadness, disgust and fear, that is, negative emotions, while they show no major difficulties in identifying the positive emotions of happiness and surprise. 
(Kennedy et al., 2012), conclude that people with autism spectrum disorder also exhibit difficulties in correctly identifying the six basic emotions that Paul Ekman put forward, an idea shared by (Aviezer et al., 2009), with the difference that the latter researcher and his team studied subjects with Huntington's disease mutations, something that was striking in this study was that most of the individuals evaluated mainly chose the emotion of disgust above the rest.

Information has also been obtained suggesting that patients with Asperger Syndrome do not show major differences with respect to healthy people in identifying emotions through facial expressions when they do so while viewing dynamic materials; however, when they perform this task while viewing static materials, their level of assertiveness drops considerably (Katsyri et al., 2008).

If we focus on another disorder such as Schizophrenia, authors such as (Dyck et al., 2010), point out that individuals with this disease have difficulties in correctly recognising emotions through facial expressions regardless of the type of materials they perceive as dynamic or static, following this line (Silver et al., 2009), share the conclusion of the article cited above, however it is worth mentioning that in this research they only analysed happiness and sadness, something that is striking is that these people when looking at a neutral face refer to observe any type of emotion.

A study that evaluated different pathologies at the same time (Alzheimer's disease, mild cognitive impairment, frontotemporal dementia and a healthy control group), found that there were no differences in the groups studied when identifying happiness and surprise emotions, however, this study found that patients with frontotemporal dementia performed worse in identifying negative emotions compared to the other three control groups (Park et al., 2017), following the same line (Elferink et al., 2015), object to the previous conclusion as they point out that people with Alzheimer's disease (AD) perform worse than people with mild cognitive impairment (MCI) and healthy people when it comes to recognising emotions through facial expressions, be they positive or negative.

On the other hand (Sato et al., 2002), point out that a person with bilateral damage to the amygdala in comparison with a person with brain damage and another healthy person, shows worse results in recognising emotions, mainly fear, noting that this type of patient tends to confuse fear and anger with happiness.

When analysing how depression interferes in the recognition of emotions through facial expressions (Kan et al., 2004 \& Surguladze et al., 2004), they mention that both depressive and nondepressive people obtain similar results performing this activity, however, they mention that people who suffer from this disease when evaluating emotions in neutral faces were inclined to determine that these expose some kind of negative emotion, this last idea is supported by (Bourke et al., 2010), 2010), but the latter authors in turn explain that depressive people more easily and assertively identify the emotion 
sadness, adding that these types of patients have a tendency to see negative emotions in facial expressions that suggest a positive emotion.

(Csukly et al., 2008), together with their research team hypothesise that the lack of adequate emotion recognition through facial expressions of people in their peers could be indicative of some kind of psychological distress or psychiatric level pathology.

While (Donadon et al., 2014), suggest that alcoholics demonstrate more problems in assertively recognising the emotions of sadness and disgust, these authors also point out that these individuals require a greater amount of time to identify fear and anger compared to the healthy control group; along the same lines (Foisy et al., 2007), conclude that alcoholism leads sufferers to observe negative emotions mainly sadness when carrying out this task.

(Dawel et al., 2012), mention that people who present psychopathy expose difficulties in identifying both positive and negative emotions and not only fear and sadness as exposed by other articles, under this same premise (Hansen et al., 2008), allude that these subjects can identify more easily the facial expression generated by the disgust, while (Pham et al., 2010), after studying a group of criminal psychopaths, another criminal group but not psychopaths and a control group with no criminal record, state that the two criminal groups show difficulties in correctly identifying emotions compared to the healthy control group.

In terms of age, authors such as (Lambrecht et al, 2012; Beer et al, 2015; Sullivan et al, 2004; \& Ruffman et al, 2008) conclude that older adults make more mistakes than younger adults when recognising the six basic emotions proposed by Ekman through facial expressions, regardless of the type of stimulus used, dynamic or static, while (Hayes et al., 2020), report that older people, compared to their younger peers, show greater facility in correctly identifying negative emotions, especially disgust.

Under this same premise (Montagne et al., 2007), explain that the (AM) obtain worse results identifying the emotions happiness, anger, fear and sadness than the (AJ), except in disgust and surprise where the two groups obtained similar results, while (Abbruzzese et al., 2019), conclude that the (AM) make more mistakes than the (AJ) when correctly identifying the emotions presented, especially fear, 2019), conclude that the (AM) make more errors than the (AJ) when correctly identifying the emotions that are presented to them, especially fear, while (Mienaltowski et al., 2013), determine that the (AM) in comparison with the (AJ) certainly identify less accurately the negative emotions fear and disgust, however, they mention that there is not a big difference between the two groups when recognising anger and sadness.

(Circelli et al., 2013), together with their team, share the idea that the (AM) have more difficulties than the (AJ) to correctly identify the emotion of fear, but they in turn refute the other conclusion reached 
by (Mienaltwski et al., 2013), since for them the (AM) identify disgust much better than the other emotions, while (Murphy et al., 2010), say that older adults show greater difficulty in correctly identifying the emotions of anger, fear and sadness.

On the other hand (Holland et al, 2018), point out that both (AM) and (AJ) do not exhibit major differences when correctly identifying emotions through facial expressions when using dynamic materials, a concept contradicted by (Beer, 2015), who mention that no matter the type of material observed, dynamic or static (AM) show worse results performing this task compared to their younger peers, (Murphy, 2010), on the other hand points out that (AM) have more problems identifying anger than $(\mathrm{AJ})$.

(West et al., 2012), explain that the (AM) have greater difficulty recognising the emotions of fear, anger and to a greater extent sadness, sharing the idea with other authors cited above that these people recognise disgust more easily, this study concludes by pointing out that both the (AM) and the (AJ) do not show major differences when it comes to assertively recognising the emotions happiness and surprise, an idea refuted by (Saito et al., 2020), who report that the (AM) do exhibit greater difficulties and make more mistakes than the $(\mathrm{AJ})$ in identifying happiness.

In reference to how the sex of the person can influence the recognition of emotions through facial expressions, research such as those of (Donges et al., 2012; Abbruzzese et al., $2019 \&$ Santos et al., 2015), conclude that women seem to obtain better results than their male peers in correctly identifying the six basic emotions described by Ekman, mainly positive emotions, however, the latter authors conclude by saying that there is still not enough validated scientific evidence or standardised methods to say which gender can perform better in the execution of this activity.

\section{DISCUSSION AND CONCLUSION:}

Ultimately, among the main findings of this systematic review, we identified that culture can modify the facial expressions that a person exhibits when feeling an emotion (Mishra et al., 2018). It highlights the possibility that there may be a modification in a person's facial expressions depending on whether the emotion they are feeling is real or acted out (Tarnowski et al., 2017).

On the other hand, it is mentioned that a person's own facial features can be confused with the features generated by an emotion, which could lead to errors when trying to recognise them (Said et al., 2009). It is detailed that the age of the individual can lead to errors being made when carrying out this task, as an older face is related to a greater extent with positive emotions (Hass et al., 2015). It is noted that even technology cannot yet perform this task accurately, as these softwares provide very different results depending on the type of variable they are programmed to analyse (Stöckli et al., (2017). 
In support of this theory (Rohr et al., 2012), they indicate that the recognition of a human being's emotion in his or her peers can even occur unconsciously, especially when discerning between a positive or negative emotion. Similarly, it is mentioned that the recognition of emotions through facial expressions is increased when dynamic rather than static materials are used (Calvo et al., 2016). According to the literature, it can be said that of the six basic universal emotions, the easiest to identify through facial expressions is happiness (Maher et al., 2014).

The idea is put forward that emotion recognition can be better performed when people have knowledge about specific landmarks to observe on the human face (Douglas et al., 2012). Moreover, it is emphasised how emotion recognition training not only helps to recognise the trained emotion, but also helps to improve the recognition of other emotions (Wang et al., 2018).

It is also observed how the presence of certain pathologies such as ADHD, BPD, epilepsy, depression, alcoholism, schizophrenia, psychopathy, etc., influence the recognition of emotions through facial expressions, the authors conclude that regardless of the disease that the person analysed in this article is suffering from, their level of assertiveness and interpretation is lower compared to a healthy person (Cortez et al., 2017; Domes et al., 2009; Edwards et al., 2017 \& Pham, 2010).

It can be observed that the age of the person also influences the degree of assertiveness they obtain in identifying emotions through facial expressions, concluding that older adults compared to younger adults make more mistakes in carrying out this activity (Lambrecht, 2012; Beer, 2015; Sullivan, 2004 \& Ruffman, 2008).

Finally, it is noted that women seem to have a slight advantage over men in recognising basic universal emotions with greater assertiveness, especially positive ones, although it is concluded that there is still insufficient scientific evidence and standardised methods to determine this accurately (Donges, 2012; Abbruzzese, 2019 \& Santos, 2015). 


\section{BIBLIOGRAPHY}

Abbruzzese, L., Magnani, N., Robertson, I. H., y Mancuso, M. (2019). Age and gender differences in emotion recognition. Frontiers in Psychology, 10, 1, 3-4. https://doi.org/10.3389/fpsyg.2019.02371

Adams, R. B., Y Kleck, R. E. (2005). Effects of Direct and Averted Gaze on the Perception of Facially Communicated Emotion. Emotion, 5(1), 3-5. https://doi.org/10.1037/1528-3542.5.1.3

Aviezer, H., Bentin, S., Hassin, R. R., Meschino, W. S., Kennedy, J., Grewal, S., Esmail, S., Cohen, S., y Moscovitch, M. (2009). Not on the face alone: perception of contextualized face expressions in Huntington's disease. Brain a Journal of Neurology, 132(6), 1633. https://doi.org/10.1093/brain/awp067

Barrett, L. F., Adolphs, R., Marsella, S., Martinez, A. M. y Pollak, S. D. (2019). Emotional Expressions Reconsidered: Challenges to Inferring Emotion From Human Facial Movements. Psychological Science in the Public Interest, 20(1), 1. https://doi.org/10.1177/1529100619832930

Beer, J. M., Smarr, C. A., Fisk, A. D., Rogers, W. A. (2015). Younger and older users' recognition of virtual agent facial expressions. Int. J. Human-Computer Studies, 75, 1, 5. https://doi.org/10.1016/j.ijhcs.2014.11.005

Biele, C., y Grabowska, A. (2006). Sex differences in perception of emotion intensity in dynamic and static facial expressions. Exp Brain Res, 171, 1-2. https://doi.org/10.1007/s00221-005-0254-0

Blandon-Gítlín, I, López, R. M., Masip, J., \& Fenn, E. (2017). Cognition, emotion and lying: implications for detecting deception. Anuario de Psicología Jurídica, 27(1), 95-96. https://doi.org/10.1016/j.apj.2017.02.004

Bourke, C., Douglas, K., y Porter, R. (2010). Processing of Facial Emotion Expression in Major Depression: A Review. SAGE journals, 44(8), 681-682. https://doi.org/10.3109/00048674.2010.496359

Calvo, M.G., Avero, P., Fernández-Martín, A., y Recio, G. (2016). Recognition thresholds for static and dynamic emotional faces. Emotion, 16 (8), 1186. https://doi.org/10.1037/emo0000192

Chóliz, M., y Fernández-Abascal, E. G. (2012). Recognition of Emotional Facial Expressions: the Role of Facial and Contextual Information in the Accuracy of Recognition. Psychological Reports, 110(1), 338. https://doi.org/10.2466/07.09.17.PR0.110.1.338-350

Circelli, K. S., Clark, U. S., y Cronin-Golomb, A. (2013). Visual scanning patterns and executive function in relation to facial emotion recognition in aging. Aging, Neuropsychology, and Cognition, 20(2), 148, 152. https://doi.org/10.1080/13825585.2012.675427

Cortez-Carbonell, I., y Cerić, F. (2017). Emotion recognition of facial expressions in adults with attention deficit hyperactivity disorder. Studies in Psychology, 38(1), 1, 4-5. https://doi.org/10.1080/02109395.2016.1268387

Csukly, G., Czobor, P., Simon, L., y Takács, B. (2008). Basic emotions and psychological distress: association between recognition of facial expressions and Symptom Checklist-90 subscales. Comprehensive Psychiatry, 49(2), 177-179, 181. https://doi.org/10.1016/j.comppsych.2007.09.001 
Dawel, A., O'Kearney, R., McKone, E., y Palermo, R. (2012). Not just fear and sadness: Meta-analytic evidence of pervasive emotion recognition deficits for facial and vocal expressions in psychopathy. Neuroscience and Biobehavioral Reviews, 36(10), 2288, 2291. https://doi.org/10.1016/j.neubiorev.2012.08.006

Domes, G., Schulze, L., y Herpertz, S. C. (2009). Emotion recognition in borderline personality disorder a review of the literature. Journal of Personality Disorders, 23(1), 6. https://doi.org/10.1521/pedi.2009.23.1.6

Donadon, M. F., y Osório, F, L. (2014). Recognition of facial expressions by alcoholic patients: a systematic literature review. Neuropsychiatric Disease and Treatment, 10, 1655-1656. https://doi.org/10.2147/NDT.S65376

Donges, U. S., Kersting, A., y Suslow, Thomas. (2012). Women's Greater Ability to Perceive Happy Facial Emotion Automatically: Gender Differences in Affective Priming. PLOS ONE, 7(7), 1-2. https://doi.org/10.1371/journal.pone.0041745

Douglas, M., Slessor, G., Allen, R., Darling, S., y Phillips, L. H. (2012). Processing Orientation and Emotion Recognition. Emotion, 12(1), 39-40. https://doi.org/10.1037/a0024775

Dyck, M., Habel, U., Slodczyk, J., Schlummer, J., Backes, V., Schneider, F., y Reske, M. (2008). Negative bias in fast emotion discrimination in borderline personality disorder. Psychological Medicine, 39(5), 855856. https://doi.org/10.1017/S0033291708004273

Dyck, M., Winbeck, M., Leiberg, S., Chen, Y., y Mathiak, K. (2010), Virtual faces as a tool to study emotion recognition deficits in schizophrenia. Psychiatry Research, 179(3), 247-248. https://doi.org/10.1016/j.psychres.2009.11.004

Edwards, M., Stewart. E., Palermo, R., y Lah, S. (2017). Facial Emotion Perception in Patients with Epilepsy: A Systematic Review with Meta-Analysis. Neuroscience and biobehavioral reviews, 83, 212213. https://doi.org/10.1016/j.neubiorev.2017.10.013

Ekman, P. (2016). What Scientists Who Study Emotion Agree About. Perspectives on Psychological Science, 11(1), 31. https://doi.org/10.1177/1745691615596992

Elferink, M. W., Tilborg, I. V., y Kessels, R. P. (2015). Perception of emotions in mild cognitive impairment and Alzheimer's dementia: does intensity matter. Translational Neuroscience, 6(1), 139-140. https://doi.org/10.1515/tnsci-2015-0013

Foisy, M. L., Kornreich, C., Petiau, C., Parez, A., Hanak, C., Verbanck, P., Pelc, I., y Philippot, P. (2007). Impaired emotional facial expression recognition in alcoholics: Are these deficits specific to emotional cues. Psychiatry Research, 150(1), 33-34. https://doi.org/10.1016/j.psychres.2005.12.008

Gosselin, P., Perron, M., y Beaupré, M. (2010). The Voluntary Control of Facial Action Units in Adults. Emotion, 10(2), 266, 268, 270. https://doi.org/10.1037/a0017748

Hansen, A. L., Johnsen, B. H., Hart, S., Waage, L., y Thayer, J. F. (2008), Brief communication: Psychopathy and recognition of facial expressions of emotion. Journal of Personality Disorders, 22(6), 639-641. https://doi.org/10.1521/pedi.2008.22.6.639 
Hass, N. C., Schneider, E. J., y Lim, S. L. (2015). Emotional expressions of old faces are perceived as more positive and less negative than young faces in young adults. Frontiers in Psychology, 6(1276), 1, 3. https://doi.org/10.3389/fpsyg.2015.01276

Hayes, G. S., McLennan, S. N., Henry, J. D., Phillips, L. H., Terrett, G., Rendell, P.G., Pelly, R. M., y Labuschagne, I. (2020). Task characteristics influence facial emotion recognition effects of age: a metaanalytic review. Psychology and aging, 35 (2), 295. https://doi.org/10.1037/pag0000441

Hess, U., y Thibault, P. (2009). Darwin and Emotion Expression. American Psychologist, 64(2), 120. https://doi.org/10.1037/a0013386

Holland, C. A., Ebner, N. C., Lin, T., y Samanez-Larkin, G. (2018). Emotion identification across adulthood using the Dynamic FACES database of emotional expressions in younger, middle aged, and older adults. Cognition and Emotion, 33(2), 245. https://doi.org/10.1080/02699931.2018.1445981

Horstmann, G. (2003). What Do Facial Expressions Convey: Feeling States, Behavioral Intentions, or Action Requests. Emotion, 3(2), 150, 161-162. https://doi.org/10.1037/1528-3542.3.2.150

Huang, C. L., Hsiao, S., Hwu, H. G., y Howng S. L. (2012). The Chinese Facial Emotion Recognition Database (CFERD): A computer-generated 3-D paradigm to measure the recognition of facial emotional expressions at different intensities. Psychiatry Research, 200(2), 928-929, 932. https://doi.org/10.1016/j.psychres.2012.03.038

Jack, R. E., Caldara, R., y Schyns, P. G. (2012). Internal Representations Reveal Cultural Diversity in Expectations of Facial Expressions of Emotion. Journal of Experimental Psychology: General, 141(1), 19, 20. https://doi.org/10.1037/a0023463

Kan, Y., Mimura, M., Kamijima, K., y Kawamura, M. (2004). Recognition of emotion from moving facial and prosodic stimuli in depressed patients. Neurology, Neurosurgery \& Psychiatry, 75(12), 1667. http://dx.doi.org/10.1136/jnnp.2004.036079

Katsyri, J., Saalasti, S., Tiippana, K., Wendt, L., y Sams, M. (2008). Impaired recognition of facial emotions from low-spatial frequencies in Asperger síndrome. Neuropsychologia, 46(7), 1888, 1890. https://doi.org/10.1016/j.neuropsychologia.2008.01.005

Kennedy, D. P., y Adolphs, R. (2012). Perception of emotions from facial expressions in high-functioning adults with autism. Neuropsychologia, 50(14), 3313-3314. https://doi.org/10.1016/j.neuropsychologia.2012.09.038

Lambrecht, L., Kreifelts, B., y Wildgruber, D. (2012). Age-Related Decrease in Recognition of Emotional Facial and Prosodic Expressions. Emotion, 12(3), 529-531. https://doi.org/10.1037/a0026827

Maher, Stephen., Ekstrom, T., y Chen, Y. (2014). Greater perceptual sensitivity to happy facial expression. Perception, 43(12), 1353-1354. https://doi.org/10.1068/p7806

Mao, Q., Pan, X., Zhan,Y. , y Shen, X. (2015). Using Kinect for real-time emotion recognition via facial expressions. Front Inform Technol Electron Eng, 16, 272. https://doi.org/10.1631/FITEE.1400209 
Mienaltowski, A., Johnson, E. R., Wittman, R., Wilson, A. T., Cassandra, S., y Norman, J. F. (2013). The visual discrimination of negative facial expressions by younger and older adults. Vision Research, 81, 1213, 15-16. https://doi.org/10.1016/j.visres.2013.01.006

Mishra, M. V., Ray, S. B., y Srinivasan, N. (2018), Cross-cultural emotion recognition and evaluation of Radboud faces database with an Indian sample. PLOS ONE, 13(10), 1, 3-4. https://doi.org/10.1371/journal.pone.0203959

Montagne, B., Kessels, P. C., De Hann, H. F., y Perrett, D. I. (2007). The emotion recognition task: a paradigm to measure the perception of facial emotional expressions at different intensities. Perceptual and Motor Skills, 104(2), 589, 591. https://doi.org/10.2466/PMS.104.2.589-598

Monti, G., y Meletti, S. (2015), Emotion recognition in temporal lobe epilepsy: A systematic review. Neuroscience and Biobehavioral Reviews, 282-283. https://doi.org/10.1016/j.neubiorev.2015.05.009

Murphy, N. A., y Isaacowitz, D. M. (2010). Age effects and gaze patterns in recognising emotional expressions: An in-depth look at gaze measures and covariates. Cognition and Emotion, 24(3), 436, 439. https://doi.org/10.1080/02699930802664623

Naab, P. J., y Russell, J. A. (2007). Judgments of Emotion from Spntaneous Facial Expressions of New Guineans. Emotion, 7(4), 736-738. https://doi.org/10.1037/1528-3542.7.4.736

Namba, S., Kabil, R. S., Miyatani, M., y Takashi N. (2018). Dynamic screens improve the ability to discriminate genuine and posed facial expressions of emotion. Frontiers in Psychology, 9(672), 1-3. https://doi.org/10.3389/fpsyg.2018.00672

Park, S., Kim, T., Shin, S. A., Kim, Y. K., Sohn, B. K., Park, H. J., Youn, J. H., y Lee, J. Y. (2017). Behavioral and Neuroimaging Evidence for Facial Emotion Recognition in Elderly Korean Adults with Mild Cognitive Impairment, Alzheimer's Disease, and Frontotemporal Dementia. Frontiers in Aging Neuroscience, 9(389), 1, 4. https://doi.org/10.3389/fnagi.2017.00389

Pham, T. H., y Philippot, P. (2010). Decoding of facial expression of emotion in criminal psychopaths. Journal of Personality Disorders, 24(4), 445, 449-450. https://doi.org/10.1521/pedi.2010.24.4.445

Ricciardi, L., Visco-Comandini, F., Erro, R., Morgante, F., Bologna, M., Fasano, A., Ricciardi, D., Edwards, M. J. y Kilner, J. (2017). Facial Emotion Recognition and Expression in Parkinson's Disease: An Emotional Mirror Mechanism. PLOS ONE, 12(1), 1-3. https://doi.org/10.1371/journal.pone.0169110

Rohr, M., Degner, J., y Wentura, D. (2012). Masked emotional priming beyond global valence activations. Cognition and Emotion, 26(2), 224, 241. https://doi.org/10.1080/02699931.2011.576852

Ruffman, T., Henry, J. D., Livingstone, V., y Phillips, L. H. (2008). A meta-analytic review of emotion recognition and aging: Implications for neuropsychological models of aging. Neuroscience and Biobehavioral Reviews, 32(4), 863, 867, 869. https://doi.org/10.1016/j.neubiorev.2008.01.001

Said, C. P., Sebe, N., y Todorov, A. (2009). Structural Resemblance to Emotional Expressions Predicts Evaluation of Emotionally Neutral Faces. Emotion, 9(2), 260-261. https://doi.org/10.1037/a0014681 
Saito, A., Sato, W., y Yoshikawa, S. (2020). Older adults detect happy facial expressions less rapidly. Royal Society Open Science, 7(3), 1-3. https://doi.org/10.1098/rsos.191715

Samara, A., Galway, L., Bond, R., y Wang, H. (2017). Affective state detection via facial expression analysis within a human-computer interaction context. Journal of Ambient Intelligence and Humanized Computing, 10, 2175. https://doi.org/10.1007/s12652-017-0636-8

Santos, L. F., y Osório, F. L. (2015). Influence of gender in the recognition of basic facial expressions: A critical literature review. World Journal of $W{ } P$ Psychiatry, 5(3), 342-344. https://dx.doi.org/10.5498/wjp.v5.i3.342

Sato, W., Hyniewska, S., Minemoto, K., y Yoshikawa, S. (2019). Facial Expressions of Basic Emotions in Japanese Laypeople. Frontiers in Psychology, 10(259), 1, 3, 9. https://doi.org/10.3389/fpsyg.2019.00259

Sato, W., Kubota, Y., Okada, T., Murai, T., Yoshikawa, S., y Sengoku, A. (2002). Seeing happy emotion in fearful and angry faces: qualitative analysis of facial expression recognition in a bilateral amygdaladamaged patient. Cortex, 38(5), 727, 729. https://doi.org/10.1016/S0010-9452(08)70040-6

Semyonov, O., Ziv-El, A., Krumhuber, E. G., Karasik, S., y Aviezer, H. (2019). Beyond shared signals: the role of the downward gaze in the stereotypical representation of sad facial expressions. Emotion. 1. https://doi.org/10.1037/emo0000706

Silver, H., Bilker, W., y Goodman, C. (2009). Impaired recognition of happy, sad and neutral expressions in schizophrenia is emotion, but not valence, specific and context dependent. Psychiatry Research, 169(2), 101-102, 104. https://doi.org/10.1016/j.psychres.2008.11.017

Slessor, G., Phillips, L. H., y Bull, R. (2010). Age-Related Changes in the Integration of Gaze Direction and Facial Expressions of Emotion. Emotion, 10(4), 555-556. https://doi.org/10.1037/a0019152

Stöckli, S., Schulte-Mecklenbeck, M., Borer, S., y Samson, A. C. (2017). Facial expression analysis with AFFDEX and FACET: A validation study. Behav Res, 50(4), 1446. https://doi.org/10.3758/s13428-0170996-1

Sullivan, S., y Ruffman, T. (2004). Emotion recognition deficits in the elderly. Intern. J. Neuroscience, 114(3), 403-404, 408. https://doi.org/10.1080/00207450490270901

Surguladze, S. A., Young, A. W., Senior, C., Brébion, G., Travis, M. J., y Phillips, M. L. (2004). Recognition Accuracy and Response Bias to Happy and Sad Facial Expressions in Patients With Major Depression. Neuropsychology, 18(2), 212-213. https://doi.org/10.1037/0894-4105.18.2.212

Tarnowski, P., Kolodziej, M., Majkowski, A., y Rak, R. J. (2017). Emotion recognition using facial expressions. Procedia Computer Science, 108, 1175-1177. https://doi.org/10.1016/j.procs.2017.05.025

Unoka, Z., Fodg, D., Füzy, M., y Csukly, G, (2011). Misreading the facial signs: Specific impairments and error patterns in recognition of facial emotions with negative valence in borderline personality disorder. Psychiatry Research, 189(3), 419, 421. https://doi.org/10.1016/j.psychres.2011.02.010 
Wang, Y., Zhu, Z, Chen., y Fang, F. (2018). Perceptual learning and recognition confusion reveal the underlying relationships among the six basic emotions. Cognition and Emotion, 33(4), 1, 3. https://doi.org/10.1080/02699931.2018.1491831

West, J. T., Horning, S. M., Klebe, K. J., Foster, S. M., Cornwell, R. E., Perrett, D., Burt, D. M., y Davis, H. P. (2012). Age Effects on Emotion Recognition in Facial Displays: From 20 to 89 Years of Age. Experimental Aging Research, 38(2), 147, 151-152. https://doi.org/10.1080/0361073X.2012.659997 